Wenjie Zhou $₫$

Ning Qiu

Ning Zhang

Zhounian Lai

Bo Gao

https://doi.org/10.21278/TOF.42306

ISSN 1333-1124

eISSN 1849-1391

\title{
RESEARCH ON STEADY-STATE CHARACTERISTICS OF CENTRIFUGAL PUMP ROTOR SYSTEM WITH WEAK NONLINEAR STIFFNESS
}

\begin{abstract}
Summary
Steady-state vibration response characteristics of a motion model of a centrifugal pump rotor system with weak nonlinear stiffness have been calculated by using the multiple scale method (MSM). The theoretical results were in good agreement with the numerical results. Based on the MSM and the numerical method, the effects of detuning parameter, nonlinear stiffness parameter and natural frequency on steady-state amplitude were also investigated. Finally, Lyapunov's theorem on stability in the first approximation was applied for the determination of the system's stable and unstable solution regions. The calculated results imply that the centrifugal pump rotor system with weak nonlinear stiffness exhibits typical nonlinear vibration characteristics. The variation of detuning parameter, nonlinear stiffness parameter and natural frequency can result in a jump phenomenon, and their corresponding curves present 'hard spring', 'soft spring' and 'S'-shaped amplitude characteristic, respectively. Smaller detuning parameter and natural frequency or greater nonlinear stiffness parameter are beneficial to decreasing the steady-state response amplitude. The results can provide reference for an investigation into nonlinear vibration characteristics of a centrifugal pump rotor system.
\end{abstract}

Key words: $\quad$ centrifugal pump, rotor system, steady-state characteristics, weak nonlinear stiffness, multiple scale method (MSM)

\section{Introduction}

Centrifugal pump rotor is one of the most fundamental parts in a centrifugal pump device. The dynamic analysis of the centrifugal pump rotor is important and essential for a centrifugal pump system. In order to reduce the research difficulty, a linear model has been often applied in the investigation into dynamic characteristics of a rotor system, such as critical speeds, mode shape as well as unbalanced responses, as mentioned in [1-6]. For some structures, when the vibration response is small, the linear model may be sufficient for a dynamic analysis of a centrifugal pump rotor. Once the vibration response exceeds a certain 
limit, some nonlinear phenomena will emerge and this linear model is no longer applicable. Actually, the centrifugal pump rotor system with a large lumped mass or low rigidity has richer nonlinear phenomena compared with other rotor systems, such as a large capacity double-suction centrifugal pump with a large ratio of length to diameter. In addition, higher demands on the operating conditions increase the possibility of large disturbance motion, so it is of great research value and engineering significance to study the nonlinear dynamic characteristics of the centrifugal pump rotor with nonlinear parameters.

Many studies on nonlinear dynamic characteristics of a centrifugal pump rotor have been carried out during the past few decades. Based on nonlinear vibration phenomena of a rotor system, Noah [7] pointed out the limitations of the linear vibration model and important effects of nonlinear factors on the rotor dynamics. Subsequently, Sundararajan and Noah [8] analyzed the forced vibration of a rotor system by using the shooting/arc-length continuation method and found that the periodic unbalanced force could cause aperiodic motion and chaotic motion. $\mathrm{Li}[9,10]$ established a rotor-bearing-seal coupled system including a nonlinear sealing and bearing lubrication force based on the Hamilton theory. The calculated results indicated that the coupled rotor system with a local nonlinear force presented various forms of nonlinear vibration, which would lead to severe vibration and endanger the safety of the rotor system. Chavez and Wiercigroch [11] investigated the complex dynamics and the bifurcation phenomenon of a Jeffcott rotor with a bearing clearance by means of the software TC-HAT. The study revealed that a rich variety of dynamics, including grazing-induced fold, period-doubling bifurcations and hysteresis loops could be produced by a cusp singularity. Zou [12, 13] established a coupled longitudinal-transverse dynamic model of a marine propulsion shaft and investigated the steady-state response including the superharmonic and the primary resonance by using the MSM. Based on lots of experiments, Muszynska and Bently regarded the circulating velocity as the key factor inducing the instability in a rotor system $[14,15]$. This nonlinear force model was widely used to calculate the sealing force and to predict the dynamic characteristics of a rotor system because the small disturbance limit condition could not be obeyed $[16,17]$. The numerical results exhibited rich forms of multiperiodic, quasi-periodic and chaotic motion and implied that the nonlinear sealing force had great influence on the dynamic characteristics of the rotor system. Hosseini and Khadem [18] studied the primary resonances of a rotating shaft with stretching nonlinearity and the characteristics of free vibration considering nonlinear inertia and curvature were further analyzed [19]. Vlajic [20, 21] studied torsional vibrations of a Jeffcott rotor subjected to the continuous stator contact numerically and analytically for both backward and forward whirling motions. The calculated results showed that torsional oscillations are complex under the nonlinear combined effect of lateral vibrations and frictional forces. Chasalevris and Papadopoulos [22] developed a semi-analytical simulation of a rotor-bearing system consisting of a journal bearing with finite length and a multi-segment continuous rotor and evaluated the nonlinear dynamic characteristics of this system in three different cases. Zhang $[23,24]$ studied the transient and steady-state nonlinear dynamics in a rotor-active magnetic bearing (AMB) system with the time-varying stiffness. The global bifurcations and chaotic motion obtained from the system implied that stiffness had a major impact on the rotor dynamics and the nonlinear parameters should be considered in the calculation of the rotor system.

Although the previous studies paid much attention to the nonlinear dynamic characteristics of the rotor system and many meaningful results were obtained, only the transient responses and specific rotor systems were considered. The steady-state response of a centrifugal pump rotor with nonlinear parameters, such as nonlinear stiffness, still needs further investigation. In addition, as one of the classical methods for weak nonlinear systems, the MSM has been widely used to solve the nonlinear vibration problem in the field of 
engineering research [25-27]. Based on these considerations, an isotropic centrifugal rotor model with weak nonlinear stiffness is established, the steady-state characteristics and the stability of this rotor system with different detuning parameters, nonlinear stiffness and natural frequency are determined by using the MSM. The theoretical results are in good agreement with the numerical results.

\section{Model of a centrifugal pump rotor system}

A typical centrifugal pump rotor system ignoring fluid-induced excitation is shown in Figure 1. The supported points at each end are distributed symmetrically according to the impeller cross section. The impeller of a common centrifugal pump can be regarded as a rigid body in the rotor dynamics analysis. In practice, the deformation of this rotor system is mainly determined by the unbalanced force, which is caused by the mass eccentricity of the impeller. The distance between the geometric center and the center of mass of the impeller is $r$.
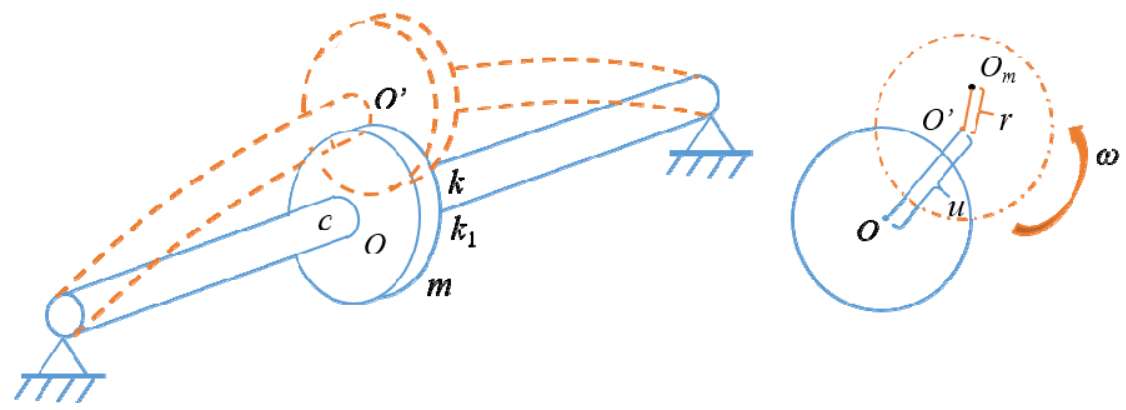

Fig. 1 Centrifugal pump rotor system

The common form of the motion equation of an unbalanced rotor system with fixed ends can be written as [28]

$$
m \ddot{u}+c \dot{u}+k u=F(t)
$$

Actually, the nonlinear elastic force of the shaft is related to the cube of displacement when large deformation occurs [29]

$$
F_{t}=k u+k_{1} u^{3}
$$

Let us assume the unbalanced harmonic force and the damping coefficient are within the same order with small parameter $\varepsilon$. Therefore, the motion equation of the rotor system considering the unbalanced harmonic force and the effect of a weak nonlinear stiffness term can be described based on Eq. (1) and Eq. (2) as follows:

$$
m \ddot{u}+\varepsilon c \dot{u}+k u+2 \varepsilon k_{1} u^{3}=\varepsilon F_{0} \cos \omega t
$$

where $F_{0}=m r \omega^{2}$.

Furthermore, Eq. (3) can be simplified as

$$
\ddot{u}+\varepsilon \xi \dot{u}+\omega_{0}^{2} u+2 \varepsilon k_{s} u^{3}=\varepsilon r \omega^{2} \cos \omega t
$$

where $\omega_{0}=\sqrt{k / m}, \xi=c / m, k_{s}=k_{1} / m . k_{\mathrm{s}}$ is related to the nonlinear stiffness of the rotor system $k_{1}$. 


\section{Solution to the motion differential equation}

\subsection{Multiple scale method (MSM)}

In order to investigate the nonlinear steady-state response of a centrifugal pump rotor system, the MSM is used to solve Eq. (4), and different scales of time variables are introduced:

$$
T_{\mathrm{n}}=\varepsilon^{n} t(n=0,1,2,3, \ldots)
$$

The first and the second derivatives of time $t$ are expressed as

$$
\begin{aligned}
& \frac{\mathrm{d}}{\mathrm{d} t}=D_{0}+\varepsilon D_{1}+\cdots \\
& \frac{\mathrm{d}^{2}}{\mathrm{~d} t^{2}}=D_{0}^{2}+2 \varepsilon D_{0} D_{1}+\cdots
\end{aligned}
$$

where $D_{n} \equiv \partial / \partial T_{n} \quad(n=0,1,2, \cdots)$.

Only the first approximate solution is considered as follows:

$$
u=u_{0}\left(T_{0}, T_{1}\right)+\varepsilon u_{1}\left(T_{0}, T_{1}\right)
$$

After substituting Eq. (8) into Eq. (4) with the power coefficient of $\varepsilon$ being equal, zeroorder and first-order approximate equations can be achieved:

$$
\begin{aligned}
& D_{0}{ }^{2} u_{0}+\omega_{0}{ }^{2} u_{0}=0 \\
& D_{0}{ }^{2} u_{1}+\omega_{0}{ }^{2} u_{1}=-2 D_{0} D_{1} u_{0}-\xi D_{0} u_{0}-2 k_{s} u_{0}^{3}+r \omega^{2} \cos \omega t
\end{aligned}
$$

The general solution to Eq. (9) can be written as

$$
u_{0}=A\left(T_{1}\right) e^{i \omega_{0} T_{0}}+\bar{A}\left(T_{1}\right) e^{-i \omega_{0} T_{0}}
$$

where $i=\sqrt{-1}, A$ and $\bar{A}$ are the conjugate complex number.

After substituting Eq. (11) into Eq. (10), the following form can be obtained for principal resonance, $\omega=\omega_{0}+\varepsilon \sigma$,

$$
D_{0}^{2} u_{1}+\omega_{0}^{2} u_{1}=\left(-2 i D_{1} A \omega_{0}-i \xi A \omega_{0}-6 k_{s} A^{2} \bar{A}+\frac{r \omega^{2}}{2} e^{i \sigma T_{1}}\right) e^{i \omega_{0} T_{0}}-2 k_{s} A^{3} e^{3 i \omega_{0} T_{0}}+C C
$$

where $C C$ stands for the complex conjugate of the preceding terms.

In order to eliminate the secular term the first term on the right-hand side of Eq. (12) must satisfy the following equation:

$$
-2 i D_{1} A \omega_{0}-i \xi A \omega_{0}-6 k_{s} A^{2} \bar{A}+\frac{r \omega^{2}}{2} e^{i \sigma T_{1}}=0
$$

Generally, the complex function A can be transformed into the exponential form as follows:

$$
A\left(T_{1}\right)=\frac{1}{2} a\left(T_{1}\right) e^{i \theta\left(T_{1}\right)}
$$


Furthermore, substituting Eq. (14) into Eq. (13), the following first-order ordinary differential equations can be obtained by separating the real and the imaginary parts:

$$
\left\{\begin{array}{l}
a^{\prime}=-\frac{1}{2} \xi a+\frac{r}{2}\left(\omega_{0}+2 \varepsilon \sigma\right) \sin \gamma \\
\gamma^{\prime}=\sigma-\frac{3}{4} \frac{a^{2} k_{s}}{\omega_{0}}+\frac{r}{2 a}\left(\omega_{0}+2 \varepsilon \sigma\right) \cos \gamma
\end{array}\right.
$$

where $\gamma=\sigma T_{1}-\theta$.

For stable motion, $a^{\prime}=\gamma^{\prime}=0$, so the non-zero constant particular solutions $a_{s}$ and $\gamma_{s}$ of Eq. (15) can be further integrated. Finally, the amplitude-frequency equation of the rotor system can be expressed as follows on the basis of the trigonometric function formula:

$$
\frac{1}{4} \xi^{2} a_{s}^{2}+\left(\sigma-\frac{3}{4} \frac{a_{s}^{2} k_{s}}{\omega_{0}}\right)^{2} a_{s}^{2}=\frac{r^{2}}{4}\left(\omega_{0}+2 \varepsilon \sigma\right)^{2}
$$

It should be noted that the amplitude-frequency equation is a sixth power function of $a_{s}$, the calculated amplitudes must be evaluated and selected according to $a_{s}>0$. The complete derivation of the analytical solution from Eq. (9) to Eq. (16) can be seen in Appendix.

\subsection{Stability analysis}

In order to evaluate the stability of the rotor system, Lyapunov's theorem on stability in the first approximation was used to determine the stability of the calculated amplitudes. The Jacobi matrix of Eq. (15), which is close to the stable points, can be obtained

$$
J=\left.\left[\begin{array}{ll}
\frac{\partial f_{1}}{\partial a} & \frac{\partial f_{1}}{\partial \gamma} \\
\frac{\partial f_{2}}{\partial a} & \frac{\partial f_{2}}{\partial \gamma}
\end{array}\right]\right|_{a=a_{s}, \gamma=\gamma_{s}}
$$

where $f_{1}=-\frac{1}{2} \xi a+\frac{r}{2}\left(\omega_{0}+2 \varepsilon \sigma\right) \sin \gamma, f_{2}=\sigma-\frac{3}{4} \frac{a^{2} k_{s}}{\omega_{0}}+\frac{r}{2 a}\left(\omega_{0}+2 \varepsilon \sigma\right) \cos \gamma$.

Therefore, the corresponding characteristic equation of Eq. (17) can be written as

$$
\left|\begin{array}{cc}
-\frac{1}{2} \xi-\lambda & -a_{s}\left(\sigma-\frac{3}{4} \frac{a_{s}^{2} k_{s}}{\omega_{0}}\right) \\
\frac{1}{a_{s}}\left(\sigma-\frac{9}{4} \frac{a_{s}^{2} k_{s}}{\omega_{0}}\right) & -\frac{1}{2} \xi-\lambda
\end{array}\right|=0
$$

By expanding the above determinant, we can obtain the quadratic equation of eigenvalues as follows:

$$
\lambda^{2}+\xi \lambda+\left(\sigma-\frac{3}{4} \frac{a_{s}^{2} k_{s}}{\omega_{0}}\right)\left(\sigma-\frac{9}{4} \frac{a_{s}^{2} k_{s}}{\omega_{0}}\right)+\frac{1}{4} \xi^{2}=0
$$


The eigenvalues of the rotor system can be solved and expressed as

$$
\lambda_{1,2}=-\frac{\xi}{2} \pm \sqrt{-\left(\sigma-\frac{3}{4} \frac{a_{s}^{2} k_{s}}{\omega_{0}}\right)\left(\sigma-\frac{9}{4} \frac{a_{s}^{2} k_{s}}{\omega_{0}}\right)}
$$

Hence the unstable condition of Eq. (20) can be concluded according to the Lyapunov's theorem on stability in the first approximation

$$
\Gamma=\xi^{2}+4\left(\sigma-\frac{3}{4} \frac{a_{s}^{2} k_{s}}{\omega_{0}}\right)\left(\sigma-\frac{9}{4} \frac{a_{s}^{2} k_{s}}{\omega_{0}}\right)<0
$$

Otherwise the steady-state motions are stable.

\section{Results and discussion}

The steady-state amplitudes calculated by the numerical method and the MSM are compared in this section. The stable region and the unstable region of the rotor system with weak stiffness are also identified. In the numerical calculation, the fourth order Runge-Kutta method is used to ensure the efficiency and accuracy of the numerical results. The main parameters and values of the rotor system are listed in Table 1 and the framework of this paper regarding steady-state characteristics is shown in Figure 2.

Table 1 Main parameters and values

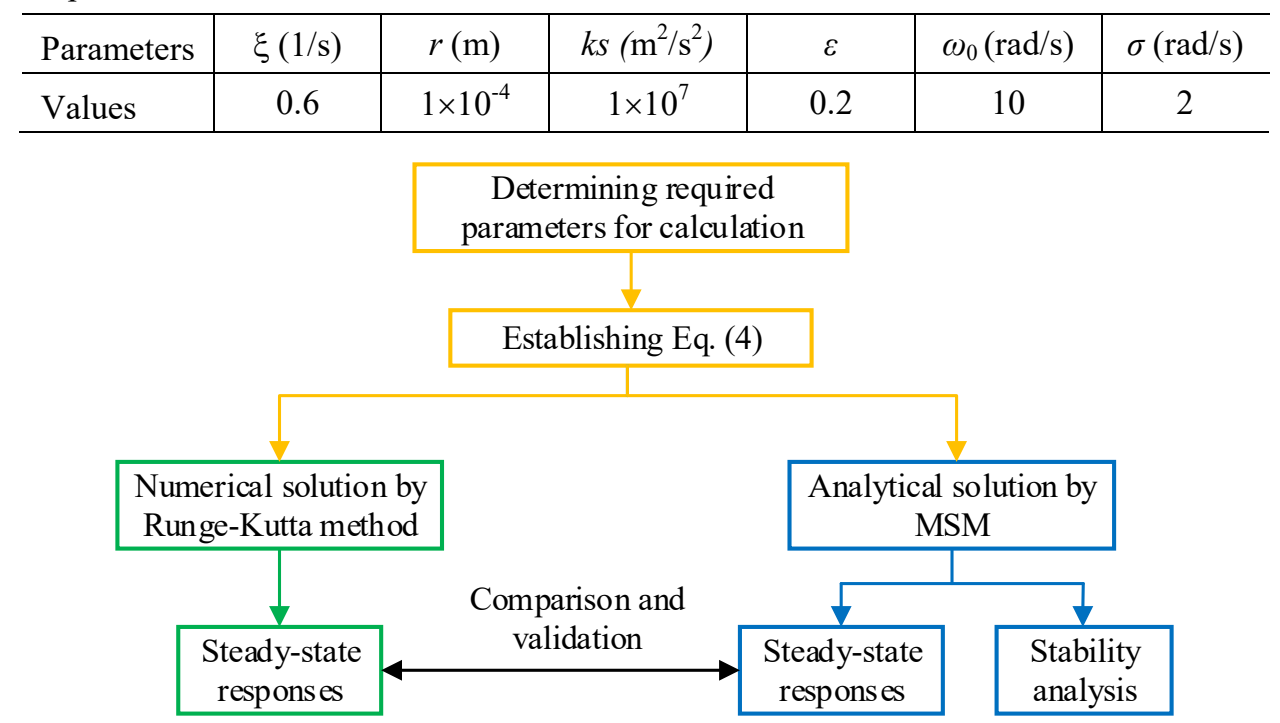

Fig. 2 Main framework for steady-state characteristics 
4.1 Influence of $\sigma$ on the steady-state amplitude

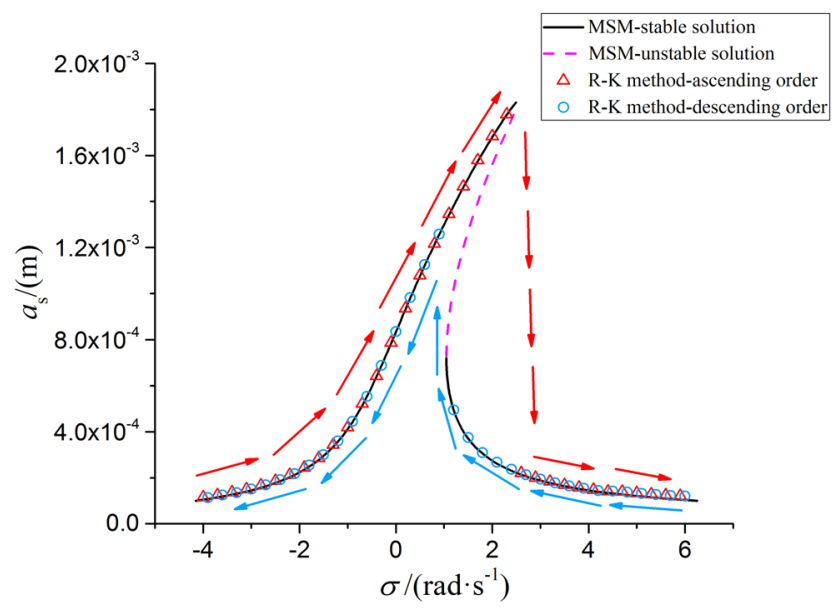

Fig. 3 Numerical and analytic results of steady-state amplitude with different $\sigma$

The steady-state amplitude of the rotor system with weak stiffness is shown in Figure 3, in which the first-order approximate numerical and analytic results are obtained for different $\sigma$. It can be seen that the analytic results calculated by the MSM are in good agreement with the numerical results. The skeleton line of the amplitude curve slants rightwards and presents typical 'hard spring' properties with an increase in the detuning parameter $\sigma$. The change rule is fundamentally different to that of the linear model, which illustrates that the weak nonlinear stiffness can cause obvious nonlinear characteristics. In addition, the jump phenomenon occurs when the detuning parameter $\sigma$ changes and the jump points do not overlap in the case of different changing orders; these results are consistent with those calculated by Eissa [30].

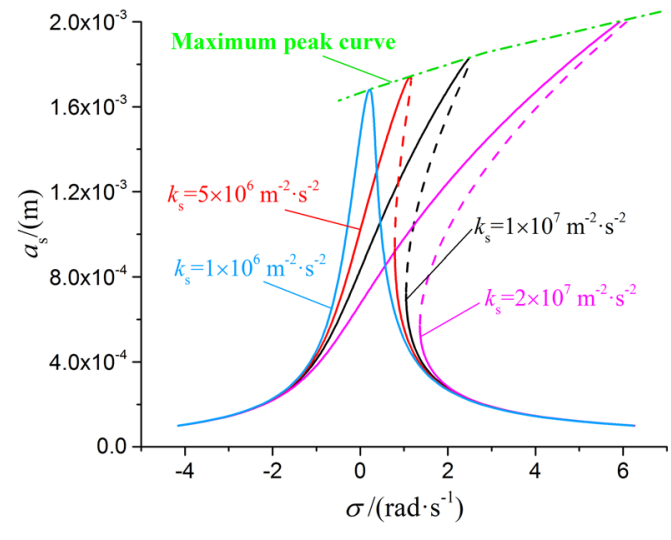

(a)

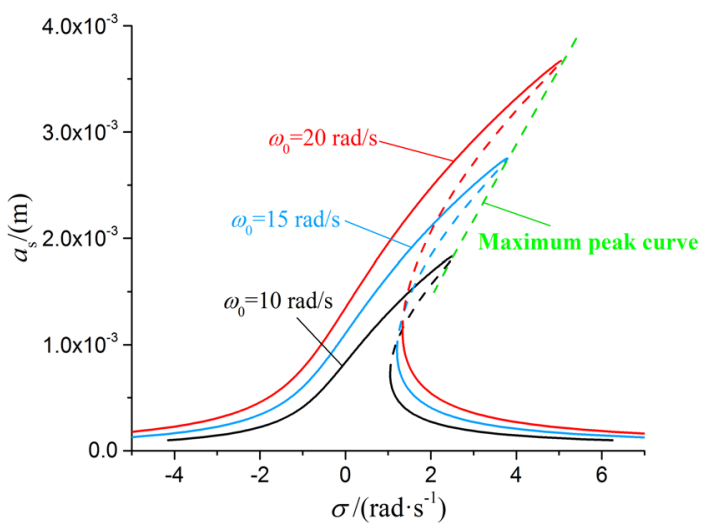

(b)

Fig. 4 Effect of $k_{\mathrm{s}}$ (a) and $\omega_{0}$ (b) on steady-state amplitude with different $\sigma$

Figure $4 \mathrm{a}$ and Figure $4 \mathrm{~b}$ show the effects of $k_{\mathrm{s}}$ and $\omega_{0}$ on analytic responses for different $\sigma$. From Figure $4 \mathrm{a}$, it can be observed that the response mode will change from single amplitude to multiple amplitudes and the maximum peak values increase as nonlinear stiffness parameter $k_{\mathrm{s}}$ increases from $1 \times 10^{6} \mathrm{~m}^{-2} \cdot \mathrm{s}^{-2}$ to $2 \times 10^{7} \mathrm{~m}^{-2} \cdot \mathrm{s}^{-2}$. In addition, the more twisted the amplitude curve is, the larger the unstable range will be. The characteristic of the steady-state amplitude curve of the unbalanced rotor system with weak stiffness is similar to that of the linear unbalanced rotor system when $k_{\mathrm{s}}$ is small and the value of the amplitude reaches the peak near the first critical speed. This phenomenon clearly implies that the larger 
nonlinear stiffness can induce more obvious nonlinear vibration characteristics and balancing the rotating shaft is necessary especially if it operates beyond the first critical speed [18]. In Figure $4 \mathrm{~b}$, the nonlinear amplitude curves exhibit a similar twisting property for different $\omega_{0}$ and the larger $\omega_{0}$ corresponds to the larger maximum amplitude, which means that the decreasing system natural frequency is beneficial to reducing the vibration amplitude. Also, the maximum peak curves in both figures are approximate linear curves.

\subsection{Influence of $k_{\mathrm{s}}$ on steady-state amplitude}

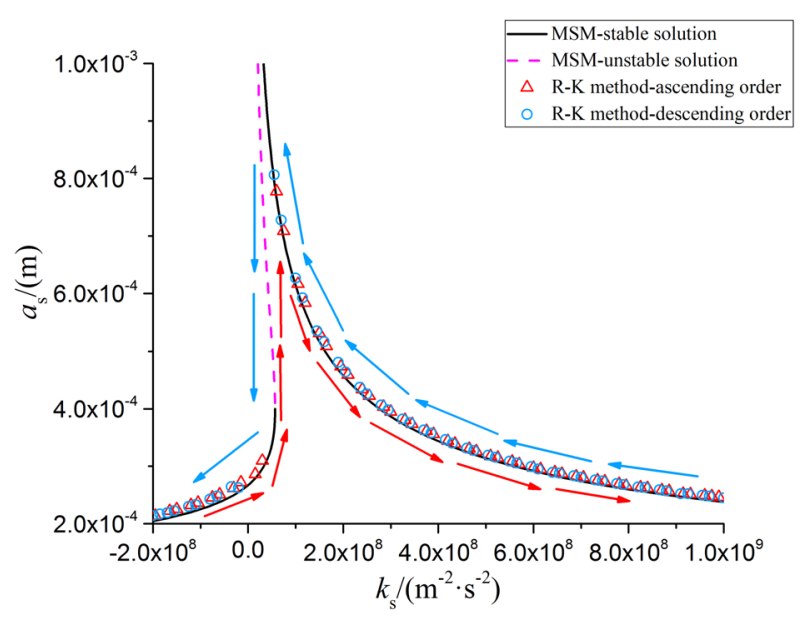

Fig. 5 Numerical and analytic results of steady-state amplitude with different $k_{\mathrm{s}}$

Figure 5 shows the first-order approximate numerical and the analytic results for different $k_{\mathrm{s}}$. The small difference between the numerical and the analytic results implies high accuracy of the calculated results. As illustrated in the figure, the skeleton line of the amplitude curve for different $k_{\mathrm{s}}$ is much different to the amplitude curve for different $\sigma$. The skeleton line slants leftwards and exhibits typical 'soft spring' properties with the change in $k_{\mathrm{s}}$. The amplitude reaches the peak when $k_{\mathrm{s}}$ is near to 0 and decreases with the increase in $k_{\mathrm{s}}$. The reason for this phenomenon is that the increase in $k_{\mathrm{s}}$ increases the total stiffness of the rotor system compared with that of the linear system. Moreover, the change in $k_{\mathrm{s}}$ can lead to strong nonlinearity and the jump phenomenon also occurs. However, the skeleton line is more vertical than in the previous cases, which indicates that the unstable solution range is sensitive to the change in $k_{\mathrm{s}}$.

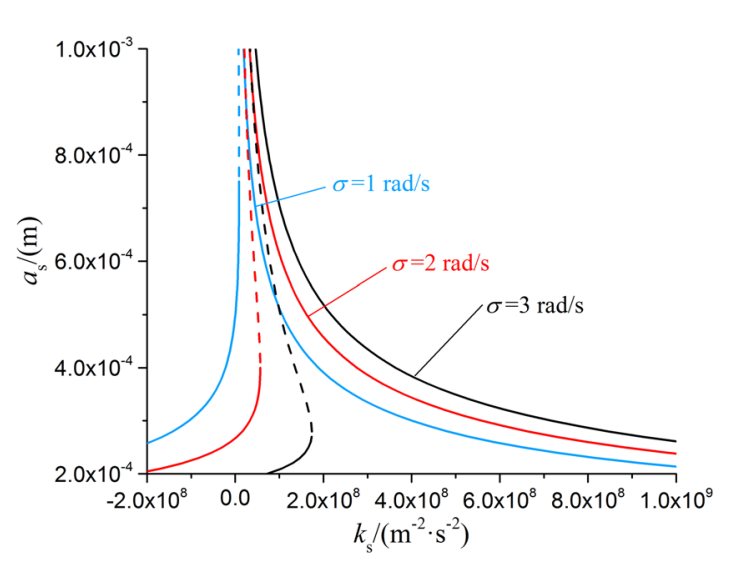

(a)

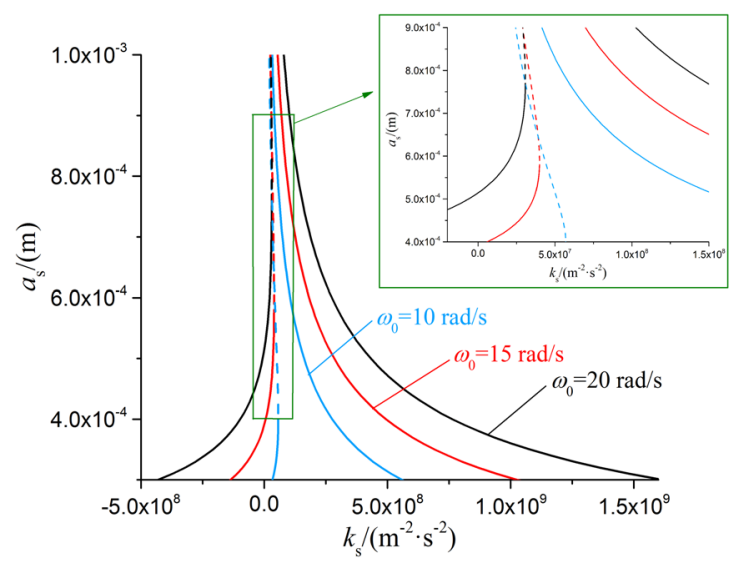

(b)

Fig. 6 Effect of $\sigma(\mathrm{a})$ and $\omega_{0}$ (b) on steady-state amplitude with different $k_{\mathrm{s}}$ 
Figure 6a shows the effect of $\sigma$ on the steady-state amplitude for different $k_{\mathrm{s}}$ when the MSM is used. It can be seen that the detuning parameter cannot change the amplitude curve form and these curves will move to the right as $\sigma$ increases, but the amplitude curve becomes most twisted when $\sigma=3 \mathrm{rad} / \mathrm{s}$. This means that smaller $\sigma$ means the excitation frequency is closer to the natural frequency and the resonance phenomenon is also more obvious when $k_{\mathrm{s}}$ approaches 0 and the larger detuning parameter has a huge influence on the nonlinearity effects. The effects of $\omega_{0}$ on the steady-state response are shown in Figure 6b. In this figure, the unstable solution curve becomes more twisted with a decrease in $\omega_{0}$ and the large natural frequency corresponds to a large changing range. This is because the excitation frequency depends linearly on the natural frequency, the increase in excitation frequency obviously enhances the unbalanced force, which induces a greater amplitude.

\subsection{Influence of $\omega_{0}$ on steady-state amplitude}

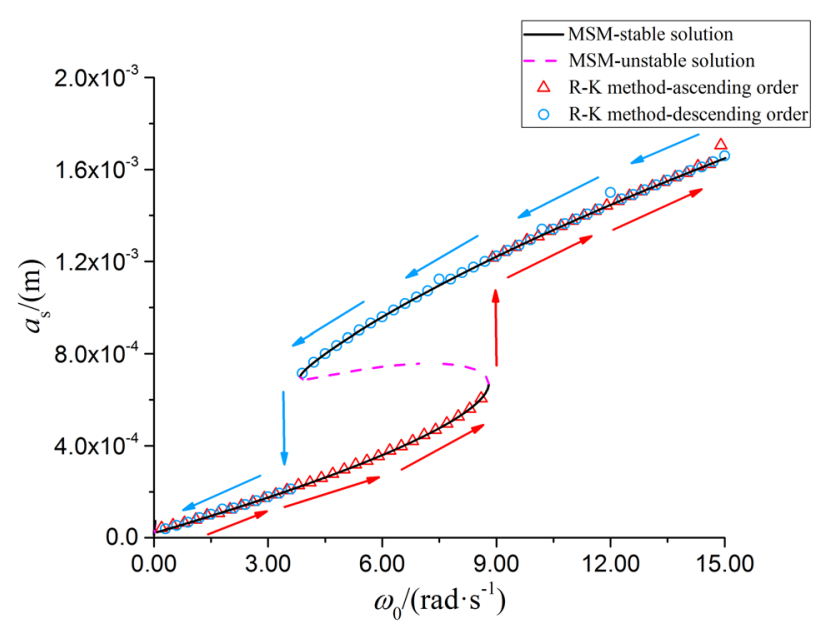

Fig. 7 Numerical and analytic results of steady-state amplitude with different $\omega_{0}$

Figure 7 shows the numerical and the analytic results of the steady-state amplitude for different natural frequency $\omega_{0}$. The numerical results are calculated by ascending and descending orders to investigate the jump phenomenon. The multi-valued curves appeared and the corresponding analytic results are divided into stable solutions and unstable solutions according to two saddle node bifurcation points. As can be seen from this figure, the changing type of the steady-state amplitude curve for different $\omega_{0}$ is completely different from that for $k_{\mathrm{s}}$ and $\sigma$. This curve presents the ' $\mathrm{S}$ ' shape instead of the rightward slant and the leftward slant form. In addition, the relationship between the amplitude and the natural frequency is approximately linear beyond the scope of the jump points. The reason for these results is that an increase in natural frequency indirectly improves the exciting force, which induces a significant increase in amplitude in the condition of primary resonance. 
W. Zhou, N. Qiu, N. Zhang, Z. Lai, B. Gao

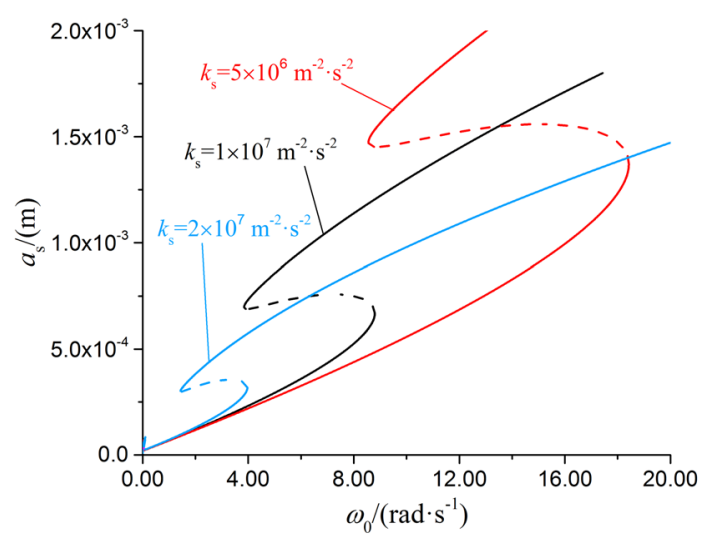

(a)
Research on Steady-State Characteristics of Centrifugal Pump Rotor System with Weak Nonlinear Stiffness

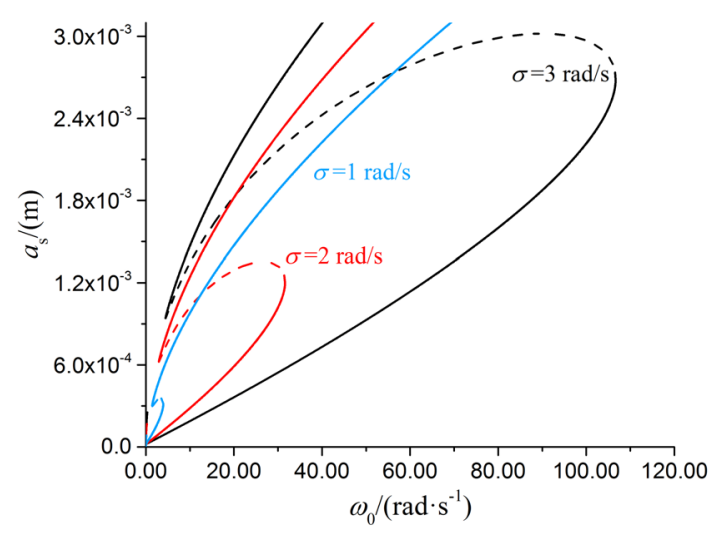

(b)

Fig. 8 Effect of $k_{\mathrm{s}}$ (a) and $\sigma(\mathrm{b})$ on steady-state amplitude with different $\omega_{0}$

Figure $8 \mathrm{a}$ and Figure $8 \mathrm{~b}$ illustrate the effects of $k_{\mathrm{s}}$ and $\sigma$ on analytic responses to different $\omega_{0}$. As shown in these figures, the value of the jump point for small $k_{\mathrm{s}}$ is the largest when the other parameters are kept unchanged. The corresponding amplitude for small $k_{\mathrm{s}}$ is also large, this is because a smaller $k_{\mathrm{s}}$ induces lower total stiffness of the rotor system. Actually, it can be known from Eq. (16) that $k_{\mathrm{s}}$ is a third power function of the steady-state amplitude $a_{\mathrm{s}}$. However, $\sigma$ has a converse effect on the steady-state amplitude, the reason for this phenomenon is that the increase in $\sigma$ will increase external excitation frequency, which is in direct proportion to the half power of the exciting force. Therefore, a small $\sigma$ presents weak nonlinear characteristics and is good for reducing the system primary resonance vibration [31]. It should be noted that the more twisted the S-shaped curve is, the more obvious the nonlinearity characteristics of the rotor system will be.

\subsection{Stability analysis}

As mentioned above, the physical parameters, detuning parameter $\sigma$, nonlinear stiffness $k_{\mathrm{s}}$ and natural frequency $\omega_{0}$, play an important part of the steady-state response of the rotor system. Hence, it is necessary to further explore the stability including different physical parameters in the stable and the unstable solution regions.

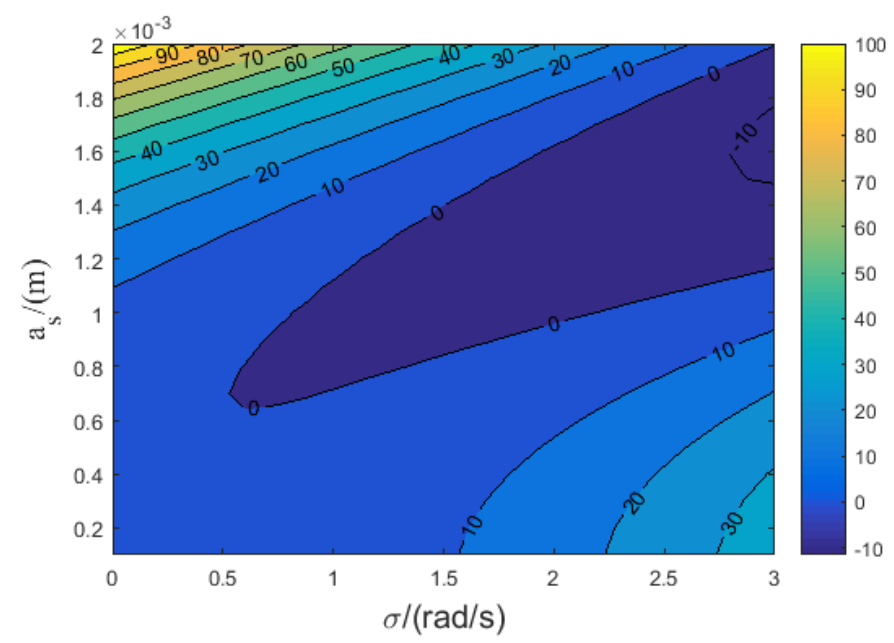

Fig. 9 Stable and unstable solution regions

The stable and the unstable solution regions of the rotor system for different $\sigma$ are shown in Figure 9. According to the results in this figure and Eq. (21), the stable solution 
regions can be determined by the contours. The motion is stable when the corresponding value $\Gamma$ exceeds 0 , otherwise the motion is unstable. Moreover, the unstable solution region is represented by the 'tongue' shape with the change in the detuning parameter [32], and the amplitude is stable when $\sigma$ is small. These calculated results verify the early inference that smaller $\sigma$ is of benefit to the steady-state vibration of the rotor system. The unstable solution region will expand as $\sigma$ increases, which is consistent with the previous results.

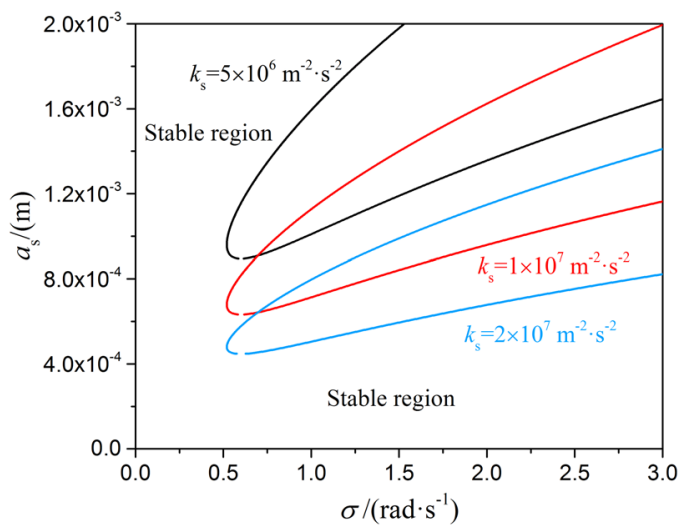

(a)

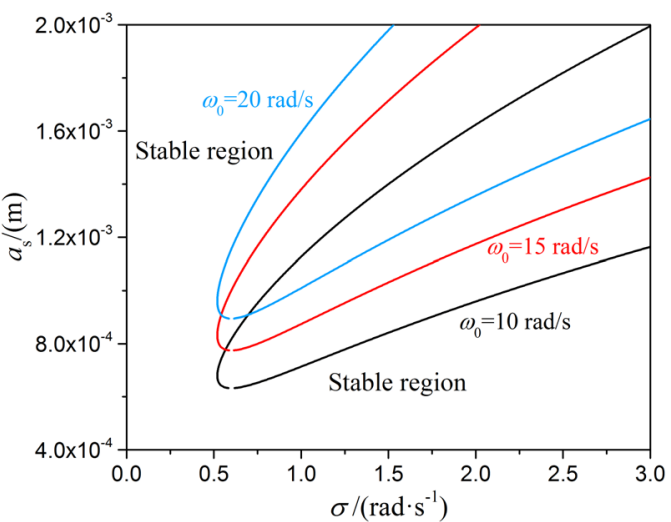

(b)

Fig. 10 Effect of $k_{\mathrm{s}}$ and $\omega_{0}$ on stable and unstable solution regions with different $\sigma$

In order to investigate the effect of $k_{\mathrm{s}}$ and $\omega_{0}$ on the stable and the unstable solution regions, the contour curve of $\Gamma=0$ in Figure 9 is extracted and the calculated results are illustrated in Figure 10. From Figure 10, the region outside the 'tongue'-shaped curve is stable, and the inside region is unstable. Changes in $k_{\mathrm{s}}$ and $\omega_{0}$ will not influence the region shape when the other parameters are kept the same. Meanwhile, the unstable region moves down and becomes narrow with the enhancement of $k_{\mathrm{s}}$, which means that a small $k_{\mathrm{s}}$ causes a large unstable amplitude. By contrast, the unstable region moves down as $k_{\mathrm{s}}$ decreases. It should be noted that the tip of every tongue is corresponding to a fixed detuning parameter. This result implies that no matter what the other parameters are, the steady-state amplitude curve of the rotor system changes from single value to multiple value when $\sigma$ is about 0.5 . All the calculated results imply that increasing $k_{\mathrm{s}}$ or reducing $\omega_{0}$ is beneficial to the stability of the rotor system with a large amplitude, and on the contrary, it has negative effect on the rotor system with a small amplitude.

\section{Conclusions}

In view of the lack of a centrifugal pump rotor model with weak nonlinear stiffness and corresponding steady-state response characteristics at present, an isotropic rotor system model for a centrifugal pump with weak nonlinear stiffness is established in order to investigate the corresponding steady-state vibration characteristics under the condition of large deformation. The steady-state amplitudes of the proposed rotor model are solved by using the numerical and the analytic method. The analytic results calculated by the MSM are in good agreement with the numerical method calculated by the R-K method. In addition, the effects of detuning parameter $\sigma$, nonlinear stiffness parameter $k_{\mathrm{s}}$ and natural frequency $\omega_{0}$ on the steady-state amplitude are also investigated so as to determine nonlinear characteristics of the rotor system. Finally, Lyapunov's theorem on stability in the first approximation is used to determine the stable and the unstable solution regions. The main results of this paper can be concluded as follows: 
1. Weak nonlinear stiffness caused by the rotor's large deformation plays an important part in the nonlinear steady-state vibration of the centrifugal pump rotor system with unbalanced force. The traditional linear rotor model is no longer suitable for the research of the weak nonlinear dynamics.

2. There exist multiple solutions and jump phenomena in the centrifugal pump rotor system with large deformation, where the stable value and the unstable value coexist in the steady-state amplitude curves.

3. The nonlinear characteristics of steady-state response curves of different physical parameters are quite different from each other, the rotor system exhibits 'hard spring', 'soft spring' and ' $\mathrm{S}$ '-shape properties with the change in $\sigma, k_{\mathrm{s}}$ and $\omega_{0}$, respectively.

4. Small $\sigma$ and $\omega_{0}$ or large $k_{\mathrm{s}}$ are beneficial to reducing the vibration amplitude of the centrifugal pump rotor system. The stable and the unstable solution regions are divided by the 'tongue'-shaped contour curve and the tip of every tongue is corresponding to a fixed $\sigma$.

\section{Acknowledgements}

This work was supported by the National Natural Science Foundation of China (Grant No. 51706087) and Priority Academic Program Development of Jiangsu Higher Education Institutions (PAPD).

\section{Nomenclature}

$\begin{array}{ll}c & \text { Damping coefficient of shaft, }(\mathrm{N} \cdot \mathrm{s} / \mathrm{m}) \\ F_{0} & \text { Unbalanced harmonic force, }(\mathrm{N}) \\ F_{t} & \text { Nonlinear elastic force of shaft, }(\mathrm{N}) \\ k & \text { Linear stiffness coefficient of shaft, }(\mathrm{N} / \mathrm{m}) \\ k_{1} & \text { Nonlinear stiffness coefficient of shaft, }\left(\mathrm{N} / \mathrm{m}^{3}\right) \\ m & \text { Mass of impeller, }(\mathrm{kg}) \\ r & \text { Eccentricity, }(\mathrm{m}) \\ t & \text { Time, }(\mathrm{s}) \\ u & \text { Displacement, }(\mathrm{m}) \\ \varepsilon & \text { Small dimensionless parameter } \\ \sigma & \text { Detuning parameter, }(\mathrm{rad} / \mathrm{s}) \\ \omega & \text { Rotating speed, }(\mathrm{rad} / \mathrm{s}) \\ \omega_{0} & \text { Natural frequency of system, }(\mathrm{rad} / \mathrm{s})\end{array}$

\section{REFERENCES}

[1] Jiang Q, Zhai L, Wang L, et al. Fluid-structure interaction analysis of annular seals and rotor systems in multi-stage pumps. Journal of Mechanical Science and Technology, 2013, 27(7): 1893-1902. https://doi.org/10.1007/s12206-013-0507-y

[2] Bonello P, Brennan M J. Modelling the dynamic behaviour of a supercritical rotor on a flexible foundation using the mechanical impedance technique. Journal of sound and vibration, 2001, 239(3): 445-466. https://doi.org/10.1006/jsvi.2000.3172

[3] Wang L, Zhou W, Wei X, et al. A coupling vibration model of multi-stage pump rotor system based on FEM. Mechanika, 2016, 22(1): 31-37. https://doi.org/10.5755/j01.mech.22.1.11420

[4] Özşahin O, Özgüven H N, Budak E. Analytical modeling of asymmetric multi-segment rotor-bearing systems with Timoshenko beam model including gyroscopic moments. Computers \& Structures, 2014, 144: 119-126. https://doi.org/10.1016/j.compstruc.2014.08.001 
Weak Nonlinear Stiffness

[5] Taplak H, Parlak M. Evaluation of gas turbine rotor dynamic analysis using the finite element method. Measurement, 2012, 45(5): 1089-1097. https://doi.org/10.1016/j.measurement.2012.01.032

[6] Koutsovasilis P. Mode shape degeneration in linear rotor dynamics for turbocharger systems. Archive of Applied Mechanics, 2017, 87(3): 575-592. https://doi.org/10.1007/s00419-016-1210-0

[7] Noah S T, Sundararajan P. Significance of considering nonlinear effects in predicting the dynamic behavior of rotating machinery, Journal of Vibration and Control, 1995, 1(4): 431-458. https://doi.org/10.1177/107754639500100403

[8] Sundararajan P, Noah S T. Dynamics of forced nonlinear systems using shooting/arc-length continuation method—application to rotor systems, Journal of Vibration and Acoustics, 1997, 119(1): 9-20. https://doi.org/10.1115/1.2889694

[9] Li W, Yang Y, Sheng D, Chen J. A novel nonlinear model of rotor/bearing/seal system and numerical analysis, Mechanism and Machine Theory, 2011, 46(5): 618-631. https://doi.org/10.1016/j.mechmachtheory.2011.01.002

[10] Li W, Yang Y, Sheng D, Chen J, Che Y. Nonlinear dynamic analysis of a rotor/bearing/seal system, Journal of Zhejiang University-Science A, 2011, 12(1): 46-55. https://doi.org/10.1631/jzus.A1000130

[11] Chávez J P, Wiercigroch M. Bifurcation analysis of periodic orbits of a non-smooth Jeffcott rotor model, Communications in Nonlinear Science and Numerical Simulation, 2013, 18(9): 2571-2580. https://doi.org/10.1016/j.cnsns.2012.12.007

[12] Zou D, Rao Z, Ta N. Coupled longitudinal-transverse dynamics of a marine propulsion shafting under superharmonic resonances, Journal of Sound and Vibration, 2015, 346: 248-264. https://doi.org/10.1016/j.jsv.2015.02.035

[13] Zou D, Liu L, Rao Z, Ta N. Coupled longitudinal-transverse dynamics of a marine propulsion shafting under primary and internal resonances, Journal of Sound and Vibration, 2016, 372: 299-316. https://doi.org/10.1016/j.jsv.2016.03.001

[14] Muszynska A, Bently D E. Frequency-swept rotating input perturbation techniques and identification of the fluid force models in rotor/bearing/seal systems and fluid handling machines, Journal of Sound and Vibration, 1990, 143(1): 103-124. https://doi.org/10.1016/0022-460X(90)90571-G

[15] Muszynska A. Improvements in lightly loaded rotor/bearing and rotor/seal models, Journal of Vibration, Acoustics, Stress and Reliability in Design. 1988, 110(2): 129-136. https://doi.org/10.1115/1.3269489

[16] Zhou W, Wei X, Wei X, Wang L. Numerical analysis of a nonlinear double disc rotor-seal system, Journal of Zhejiang University SCIENCE A, 2014, 15(1): 39-52. https://doi.org/10.1631/jzus.A1300230

[17] Ding Q, Cooper J E, Leung A Y T. Hopf bifurcation analysis of a rotor/seal system, Journal of Sound and Vibration, 2002, 252(5): 817-833. https://doi.org/10.1006/jsvi.2001.3711

[18] Hosseini S A A, Khadem S E. Analytical solution for primary resonances of a rotating shaft with stretching non-linearity, Proceedings of the Institution of Mechanical Engineers, Part C: Journal of Mechanical Engineering Science, 2008, 222(9): 1655-1664. https://doi.org/10.1243/09544062JMES923

[19] Hosseini S A A, Khadem S E. Free vibrations analysis of a rotating shaft with nonlinearities in curvature and inertia, Mechanism and Machine theory, 2009, 44(1): 272-288. https://doi.org/10.1016/j.mechmachtheory.2008.01.007

[20] Vlajic N, Champneys A R, Balachandran B. Nonlinear dynamics of a Jeffcott rotor with torsional deformations and rotor-stator contact, International Journal of Non-Linear Mechanics, 2017, 92: 102110. https://doi.org/10.1016/j.ijnonlinmec.2017.02.002

[21] Vlajic N, Liu X, Karki H, Balachandran B. Torsional oscillations of a rotor with continuous stator contact, International Journal of Non-Linear Mechanics, 2014, 83: 65-75. https://doi.org/10.1016/j.ijmecsci.2014.03.025

[22] Chasalevris A, Papadopoulos C. A novel semi-analytical method for the dynamics of nonlinear rotorbearing systems, Mechanism and Machine theory, 2014, 72: 39-59. https://doi.org/10.1016/j.mechmachtheory.2013.09.014

[23] Zhang W, Zu J W. Transient and steady nonlinear responses for a rotor-active magnetic bearings system with time-varying stiffness, Chaos, Solitons \& Fractals, 2008, 38(4): 1152-1167. https://doi.org/10.1016/j.chaos.2007.02.002

[24] Zhang W, Zu J W, Wang F X. Global bifurcations and chaos for a rotor-active magnetic bearing system with time-varying stiffness, Chaos, Solitons \& Fractals, 2008, 35(3): 586-608. https://doi.org/10.1016/j.chaos.2006.05.095 
[25] Saeed N A F A H, Kamel M. Nonlinear PD-controller to suppress the nonlinear oscillations of horizontally supported Jeffcott-rotor system, International Journal of Non-Linear Mechanics, 2016, 87: 109-124. https://doi.org/10.1016/j.ijnonlinmec.2016.10.003

[26] Silva C J, Daqaq M F. Nonlinear flexural response of a slender cantilever beam of constant thickness and linearly-varying width to a primary resonance excitation, Journal of Sound and Vibration, 2017, 389 : 438-453. https://doi.org/10.1016/j.jsv.2016.11.029

[27] Awrejcewicz J, Dzyubak L P. 2-dof non-linear dynamics of a rotor suspended in the magnetohydrodynamic field in the case of soft and rigid magnetic materials, International Journal of Non-Linear Mechanics, 2010, 45(9): 919-930. https://doi.org/10.1016/j.ijnonlinmec.2010.01.006

[28] Lee C W. Vibration Analysis of Rotors, Springer, Netherlands, 1993.

[29] Yabuno H, Kashimura T, Inoue T, Ishida Y. Nonlinear normal modes and primary resonance of horizontally supported Jeffcott rotor, Nonlinear. Dynamics, 2011, 66(3): 377-387. https://doi.org/10.1007/s11071-011-0011-9

[30] Eissa M H, Hegazy U H, Amer Y A. Dynamic behavior of an AMB supported rotor subject to harmonic excitation, Applied Mathematical Modelling, 2008, 32(7): 1370-1380. https://doi.org/10.1016/j.apm.2007.04.005

[31] Khadem S E, Shahgholi M, Hosseini S A A. Primary resonances of a nonlinear in-extensional rotating shaft, Mechanism and Machine theory, 2010, 45(8): 1067-1081. https://doi.org/10.1016/j.mechmachtheory.2010.03.012

[32] El-Bassiouny A F. Fundamental and subharmonic resonances of harmonically oscillation with time delay state feedback, Shock and Vibration, 2006, 13(2): 65-83. https://doi.org/10.1155/2006/842318

Submitted: $\quad$ 18.9.2017

Accepted: $\quad 08.5 .2018$
Wenjie Zhou*

Ning Zhang

Bo Gao

School of Energy and Power Engineering, Jiangsu University, Zhenjiang 212013, China

Ning Qiu

Research Center of Fluid Machinery

Engineering and Technology, Jiangsu University, Zhenjiang 212013, China

Zhounian Lai

Department of Civil and Environmental Engineering, the Hong Kong University of Science and Technology, Hong Kong,

China

*Corresponding author, zhouwenjiezwj@ujs.edu.cn 


\section{APPENDIX A}

Additional mathematical steps regarding the derivation of the analytical solution (Eqs. 9-16) are the following:

As the paper has shown, the zero-order and the first-order approximate equations can be obtained as follows:

$$
\begin{aligned}
& D_{0}^{2} u_{0}+\omega_{0}^{2} u_{0}=0 \\
& D_{0}{ }^{2} u_{1}+\omega_{0}{ }^{2} u_{1}=-2 D_{0} D_{1} u_{0}-\xi D_{0} u_{0}-2 k_{s} u_{0}^{3}+r \omega^{2} \cos \omega t
\end{aligned}
$$

The general solution to the zero-order approximate equation (A-1) can be written as

$$
u_{0}=A\left(T_{1}\right) e^{i \omega_{0} T_{0}}+\bar{A}\left(T_{1}\right) e^{-i \omega_{0} T_{0}}
$$

Also, the trigonometric function needs to be changed into the form of plural

$$
\cos \omega t=\frac{e^{i \omega t}+e^{-i \omega t}}{2}
$$

Substituting Eq. (A-3) and Eq. (A-4) into Eq. (A-2), the first-order approximate equation can be obtained for the principal resonance

$$
\begin{aligned}
D_{0}{ }^{2} u_{1}+\omega_{0}{ }^{2} u_{1}= & -2 i \omega_{0} D_{1}\left(A e^{i \omega_{0} T_{0}}-\bar{A} e^{-i \omega_{0} T_{0}}\right)-i \xi \omega_{0}\left(A e^{i \omega_{0} T_{0}}-\bar{A} e^{-i \omega_{0} T_{0}}\right)-2 k_{s}\left(A^{3} e^{3 i \omega_{0} T_{0}}\right. \\
& \left.+3 A^{2} \bar{A} e^{i \omega_{0} T_{0}}+3 A \bar{A}^{2} e^{-i \omega_{0} T_{0}}+\bar{A}^{3} e^{-3 i \omega_{0} T_{0}}\right)+\frac{r \omega^{2}}{2}\left[e^{i\left(\omega_{0} T_{0}+\sigma T_{1}\right)}+e^{-i\left(\omega_{0} T_{0}+\sigma T_{1}\right)}\right]
\end{aligned}
$$

Considering the conjugated structure, the simplified form of Eq. (A-5) can be written as

$$
D_{0}^{2} u_{1}+\omega_{0}^{2} u_{1}=\left(-2 i D_{1} A \omega_{0}-i \xi A \omega_{0}-6 k_{s} A^{2} \bar{A}+\frac{r \omega^{2}}{2} e^{i \sigma T_{1}}\right) e^{i \omega_{0} T_{0}}-2 k_{s} A^{3} e^{3 i \omega_{0} T_{0}}+C C
$$

where $C C$ is the complex conjugate term.

It needs to be noted that the first term on the right-hand side of Eq. (A-6) is secular term, which must satisfy the following equation:

$$
-2 i D_{1} A \omega_{0}-i \xi A \omega_{0}-6 k_{s} A^{2} \bar{A}+\frac{r \omega^{2}}{2} e^{i \sigma T_{1}}=0
$$

Therefore, the following differential equation can be obtained from Eq. (A-7):

$$
D_{1} A=\frac{i}{2 \omega_{0}}\left(i \xi A \omega_{0}+6 k_{s} A^{2} \bar{A}-\frac{r \omega^{2}}{2} e^{i \sigma T_{1}}\right)
$$

Generally, the complex function $A$ can be expressed as

$$
A\left(T_{1}\right)=\frac{1}{2} a\left(T_{1}\right) e^{i \theta\left(T_{1}\right)}
$$

Furthermore, substituting Eq. (A-9) into Eq. (A-8), the following equation can be obtained: 


$$
\frac{1}{2} a^{\prime} e^{i \theta}+\frac{1}{2} i \theta^{\prime} a e^{i \theta}=\frac{i}{2 \omega_{0}}\left(\frac{i}{2} \xi a \omega_{0} e^{i \theta}+\frac{3}{4} a^{3} k_{s} e^{i \theta}-\frac{r \omega^{2}}{2} e^{i \sigma T_{1}}\right)
$$

Therefore, the further first-order ordinary differential equations can be obtained by separating the real and the imaginary parts:

$$
\left\{\begin{array}{l}
a^{\prime}=-\frac{1}{2} \xi a+\frac{r \omega^{2}}{2 \omega_{0}} \sin \left(\sigma T_{1}-\theta\right) \\
\theta^{\prime}=\frac{3}{4} \frac{a^{2} k_{s}}{\omega_{0}}-\frac{r \omega^{2}}{2 a \omega_{0}} \cos \left(\sigma T_{1}-\theta\right)
\end{array}\right.
$$

In order to simply Eq. (A-11), the following equation is introduced:

$$
\gamma=\sigma T_{1}-\theta
$$

Therefore, Eq. (A-11) can be transformed into

$$
\left\{\begin{array}{l}
a^{\prime}=-\frac{1}{2} \xi a+\frac{r}{2}\left(\omega_{0}+2 \varepsilon \sigma\right) \sin \gamma \\
\gamma^{\prime}=\sigma-\frac{3}{4} \frac{a^{2} k_{s}}{\omega_{0}}+\frac{r}{2 a}\left(\omega_{0}+2 \varepsilon \sigma\right) \cos \gamma
\end{array}\right.
$$

For stable motion, $a^{\prime}=\gamma^{\prime}=0$, so the non-zero constant particular solutions $a_{s}$ and $\gamma_{s}$ of Eq. (A-13) can be further integrated:

$$
\left(\frac{1}{2} \xi a_{s}\right)^{2}+\left(\sigma a_{s}-\frac{3}{4} \frac{a_{s}^{3} k_{s}}{\omega_{0}}\right)^{2}=\left[\frac{r}{2}\left(\omega_{0}+2 \varepsilon \sigma\right) \sin \gamma_{s}\right]^{2}+\left[\frac{r}{2}\left(\omega_{0}+2 \varepsilon \sigma\right) \cos \gamma_{s}\right]^{2}
$$

Finally, the following amplitude-frequency equation of the rotor system can be obtained on the basis of the trigonometric function formula from Eq. (A-14)

$$
\frac{1}{4} \xi^{2} a_{s}^{2}+\left(\sigma-\frac{3}{4} \frac{a_{s}^{2} k_{s}}{\omega_{0}}\right)^{2} a_{s}^{2}=\frac{r^{2}}{4}\left(\omega_{0}+2 \varepsilon \sigma\right)^{2}
$$

